\title{
174. NUEVOS TÁXONES PARA LA FLORA DE LA MONTAÑA PALENTINA (ESPAÑA)
}

\author{
$\mathrm{M}^{\mathrm{a}}$ Barbara ARU ${ }^{1}$, Elena de $\mathrm{PAZ}^{2}$, Ruth MARTÍNEZ ${ }^{3}$,
} Raquel ALONSO ${ }^{1}$ y Marta Eva GARCÍA ${ }^{1 *}$

Recibido el 16 de abril de 2008, aceptado para su publicación el 4 de julio de 2008

Publicado «on line» en julio de 2008

New taxa in Palencia's Mountain flora (Spain)

Palabras clave. Corología, taxon, plantas vasculares, Palencia, España.

Key words. Chorology, taxa, vascular plants, Palencia, Spain.

En este trabajo se aportan citas de plantas que, en la mayoría de los casos, constituyen novedades provinciales para Palencia o bien su presencia es muy escasa en el territorio que nos ocupa. Todos los táxones han sido herborizados en la zona norte de la provincia, concretamente en el Parque Natural de Fuentes Carrionas y Fuente Cobre - Montaña Palentina (fig.1).

Los pliegos correspondientes se encuentran depositados en el Herbario LEBJaime Andrés Rodríguez de la Universidad de León. A continuación se relacionan por orden alfabético y se detallan los siguientes datos: localidad, coordenadas UTM, altitud, ecología, fecha de recolección, colectores y número de registro en el herbario.

\section{Anagallis tenella (L.) L.}

PALENCIA: Salcedillo, 30TUN939574, 1360 m, aulagar-tojal, 30-IX-2006, B. Aru, LEB 92318.

Taxon muy frecuente en las provincias limítrofes (Asturias, Cantabria, Burgos, León); sin embargo, en Palencia únicamente ha sido citado en dos localidades, Aguilar, hacia Valberzoso y La Pernía, Camasobres (Aedo et al., 1997 y 2001).

Aquilegia pyrenaica DC. subsp. discolor (Levier \& Leresche) Pereda \& M. Laínz

PALENCIA: Valle de Pineda, El Arenal, 30TUN667588, $1658 \mathrm{~m}$, pizarras calcáreas de la Formación Vidrieros, 24-VII-2007, B. Aru \& A.F. Salegui, LEB 92320.

No se ha encontrado ninguna cita para Palencia en la bibliografía consultada.

\section{Aster alpinus L.}

PALENCIA: Peña Labra, 30TUN833669, $1810 \mathrm{~m}$, pastizal pedregoso calizo, 03-IX-2006, B. Aru, LEB 92321. Lores, 30TUN692623, 1780 m, pastizal pedregoso calizo, 14-VII-2007, B. Aru \& A.F. Salegui, LEB 92317.

Primeras localidades de la provincia de Palencia donde ha sido hallado este taxon, aunque existen varias citas de Picos de Europa en provincias limítrofes (Nava, 1988).

\section{Circaea lutetiana L. subsp. lutetiana}

PALENCIA: Piedrasluengas, 30TUN805670, 1079 m, hayedo, 12-VIII-2006, B. Aru, LEB 92323.

Novedad provincial de una planta abundante en el resto de la comunidad de Castilla y León,

Este trabajo ha sido realizado en el marco del Proyecto de Investigación "Cartografía Detallada de Hábitats del Anexo I de la Directiva 92/43/CEE a escala 1:10.000 en Diversos Espacios Incluidos en la Red Natura 2000", contrato suscrito con la Universidad de Salamanca para realizar dicho proyecto en el Parque Natural de Fuentes Carrionas y Fuente Cobre - Montaña Palentina. 


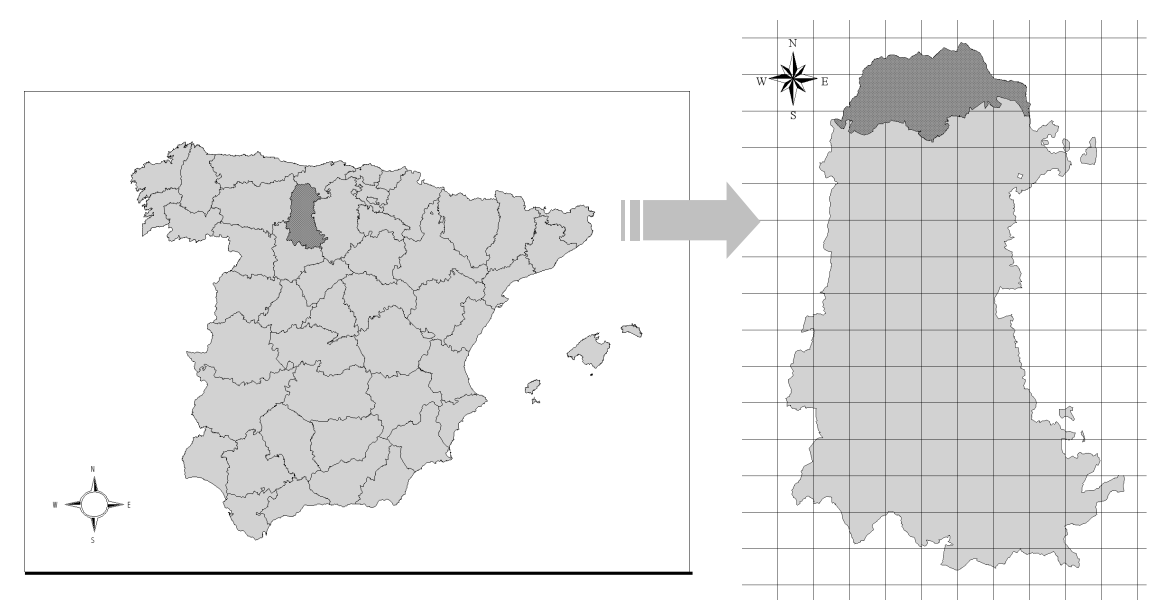

Figura 1. Situación del Parque Natural en España peninsular y en la provincia de Palencia.

así como en las limítrofes Asturias y Cantabria.

Cymbalaria muralis P. Gaertn., B. Mey. \& Scherb.

PALENCIA: Escudo de Curavacas, 30TUN629618, $1783 \mathrm{~m}$, en paredón rezumante, 19VIII-2007, B. Aru, LEB 92315.

Taxon relativamente abundante en la Península Ibérica; sorprende su escasa presencia en esta provincia y concretamente en la montaña palentina. Ha sido citado de Cervera de Pisuerga y Aguilar de Campoo (Losa España, 1942 y 1957).

Festuca rivas-martinezii Fuente \& Ortúñez subsp. rectifolia Fuente, Ortúñez \& Ferrero PALENCIA: Colonia Sierra del Brezo, 30TUN507466, 1245 m, brezal, 10-VII-2003, R. Martínez, M.E. García \& R. Alonso, LEB 92313.

Aunque en la descripción original de este endemismo ibérico (Fuente, Ortúñez \& Ferrero, 1997) se incluye el norte de Palencia en su área de distribución, no se cita el taxon de ninguna localidad concreta ni se indica material alguno de herbario. No hemos hallado ninguna otra mención del mismo en la bibliografía consultada.

Festuca rivas-martinezii Fuente \& Ortúñez subsp. rivas-martinezii

PALENCIA: Velilla del Río CarriónCervera de Pisuerga. Desviación hacia Pico
Orvillo, 30TUN526495, prado, 31-V-2003, R. Martínez, M.E. García \& R. Alonso, LEB 92314.

Endemismo ibérico especialmente frecuente en el Sistema Central, no ha sido colectado, hasta ahora, en la provincia de Palencia.

Fritillaria legionensis Llamas \& Andrés (fig.2)

PALENCIA: Lores, 30TUN736610, 1298 $\mathrm{m}$, claro de hayedo, 19-V-2007, B. Aru, LEB 92319.

Únicamente se conocen citas de este taxon en la provincia de León (Llamas \& Andrés, 1983). El ejemplar que se reseña forma parte de la única población encontrada en la montaña palentina. Aparece en La Lista Roja de la Flora Vascular Española con la categoría VU, criterio D2 (VV. AA., 2000).

\section{Juncus fontanesii J.Gay}

PALENCIA: Vidrieros, 30TUN638567 1340 m, sauceda-fresneda, 23-VII-2007, B. Aru, LEB 92316. San Salvador de Cantamuda, 30TUN784562, $1080 \mathrm{~m}$, sustrato silíceo, 6-VIII2006, B. Aru, LEB 86729.

Novedad provincial que, además, es interesante bajo el punto de vista biogeográfico puesto que, con estas citas, se amplía su área de distribución hacia la zona noroccidental ibérica, donde no hemos hallado constancia de su presencia. Las citas más próximas corresponden 


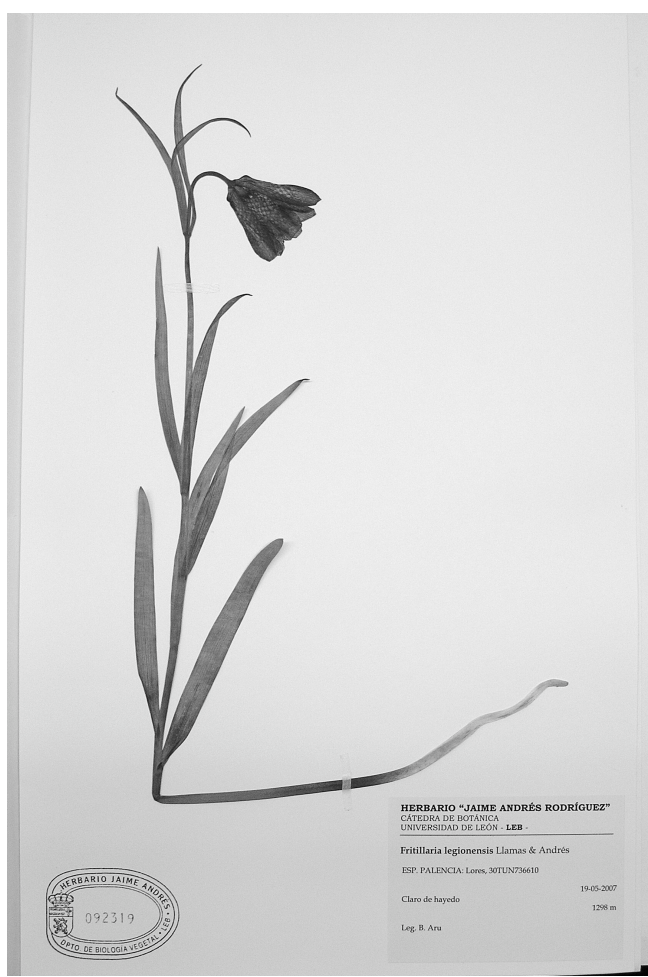

Fig. 2.- Fritillaria legionensis Llamas \& Andrés. LEB 92319. Palencia, Lores, en claro de hayedo.

a las provincias de Burgos (Fernández Carvajal, 1978 y Navarro Sánchez, 1976) y Valladolid (Fernández Alonso, 1985).

Linaria alpina (L.) Mill.subsp. alpina

PALENCIA: Curavacas, 30TUN638589, 2070 m, canchal, 23-VII-2007, B. Aru, LEB 88789.

Taxon abundantemente citado en la comunidad de Castilla y León que, en Palencia, solamente ha sido mencionado de Velilla del Río Carrión, en la subida desde Cardaño de Arriba al Pozo de las Lomas, y en La Peña del Fraile (Montserrat, 1994). La mayoría de las menciones lo son a nivel específico.

Petasites hybridus (L.) G.Gaertn., B.Mey. \& Scherb. (fig.3)

PALENCIA: Lores, 30TUN7561, 1100 m,

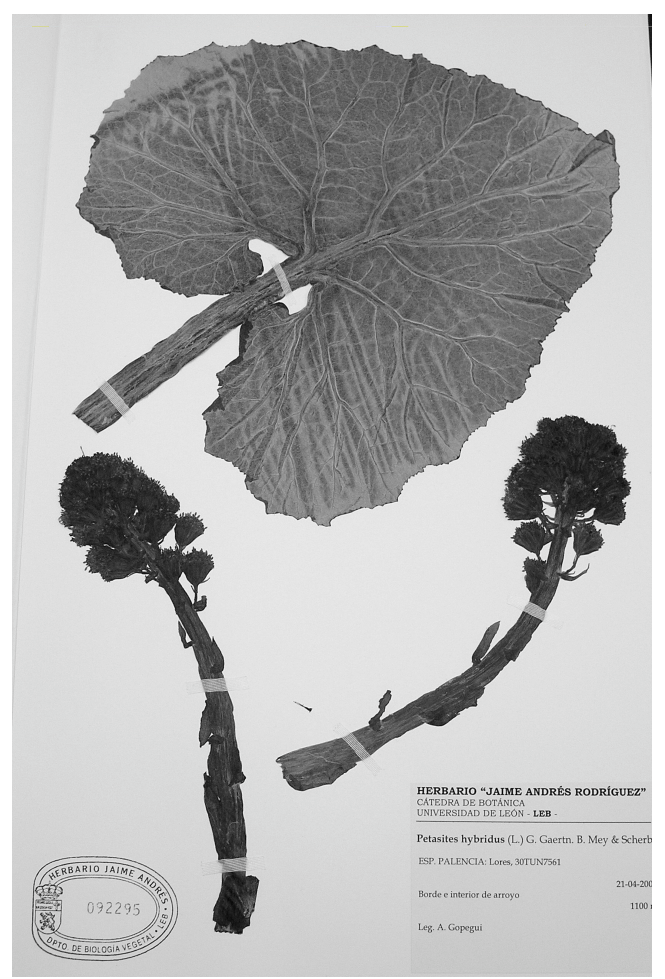

Fig. 3.- Petasites hybridus (L.) G. Gaertn., B. Mey \& Scherb. LEB 92295. Palencia, Lores, en arroyo.

borde e interior de arroyo, 21-IV-2005, A. Gopegui, LEB 92295.

Novedad provincial de la que se conoce una sola mención en Burgos (Willkomm, M. \& J. Lange, 1861-1880) en la comunidad de Castilla y León. Es un taxon de distribución fundamentalmente pirenaica.

Quercus orocantabrica Rivas Mart., Penas, Díaz \& Llamas

PALENCIA: Pico Orvillo. La Tuda, 30TUN515512, 1665 m, en comunidad de Rumex suffruticosus y Vaccinium myrtillus, 6-VIII-2003, R. Martínez, M.E. García \& R. Alonso, LEB 92294. Valle de Pineda. Las Lagunillas, 30TUN675575, $1630 \mathrm{~m}$, pedreras y canchales cuarcíticos, 23VIII-2007, B. Aru, LEB 92293. Valle de Pineda. Valcerezo, 30TUN694559, $1550 \mathrm{~m}$, ladera pedregosa, 23-VIII-2007, B. Aru, LEB 92292. 
Vidrieros, 30TUN655554, $1990 \mathrm{~m}$, pedregal silíceo, 23-VIII-2007, B. Aru, LEB 92312.

Hasta ahora, este roble había sido citado únicamente en la provincia de León, donde fue descrita la especie (Puerto de Las Señales, RivasMartínez \& al, 2002). Nuestras citas constituyen las primeras menciones del taxon en localidades de otra provincia, lo que puede ser indicativo de una mayor presencia en la Cordillera Cantábrica. Además de los pliegos arriba reseñados, hemos localizado el taxon en Salcedillo, en el límite con Cantabria, en laderas pedregosas, 30TUN974574.

Ranunculus alpestris L. subsp. leroyi M.Laínz PALENCIA: Espigüete (cara norte), 30TUN549564, $2280 \mathrm{~m}$, suelo pedregoso calizo quionófilo, 26-VIII-2004, R. Alonso, V. Castro \& C.R. Fuertes, LEB 92324.

Constituye la primera mención de la subespecie para Palencia. G. López González (in Castroviejo, 1986) reconoce su posible existencia en esta provincia pero sin respaldo de citas bibliográficas ni pliegos de herbario. Como Ranunculus alpestris ha sido citada en Velilla del Río Carrión, en la umbría del Espigüete (Laínz, 1960 y Losa España, 1957).

\section{Rubus brigantinus Samp.}

PALENCIA: La Mata, Arroyo del Monte, 30TUN692486, 1166 m, espinar, 29-VI-2003, R. Martínez, M.E. García \& R. Alonso, LEB 92297.

Según E. Monasterio-Huelin (in Castroviejo, 1998) este taxon se distribuye fundamentalmente por el noroeste de Portugal. En España se conocía de dos localidades solamente: Los Condemios (Guadalajara) y proximidades de Velilla del Río Carrión, (Palencia). Nuestra cita de La Mata constituye, por tanto, la segunda mención provincial.

AgRADECIMIENTOS. A Víctor Castro González y Antonio Gopegui, por su colaboración en la herborización de algunos táxones.

\section{BIBLIOGRAFÍA}

AEDO, C., J.J. ALDASORO, J.M. ARGÜELLES, J.L. DÍAZ, A. DÍEZ, J.M. GONZÁLEZ, M. LAÍNZ, G. MORENO, J. PATALLO y O.
SÁNCHEZ -1997- Contribuciones al conocimiento de la flora cantábrica, III. Anales Jard. Bot. Madrid 55(2): 321-350.

AEDO, C., J.J. ALDASORO, J.M. ARGÜELLES, L. CARLÓN, A. DÍEZ, J.M. GONZÁLEZ, M. LAÍNZ, G. MORENO, J. PATALLO \& O. SÁNCHEZ -2001- Contribuciones al conocimiento de la flora cantábrica, IV. Bol. Cien. Nat. R.I.D.E.A. 46: 7-119.

FERNÁNDEZ ALONSO, J.L. -1985- Flórula del término municipal de Encinas de Esgueva y zonas limitrofes. Tesis de Licenciatura. Fac. Biología. Univ. Salamanca.

FERNÁNDEZ-CARVAJAL, M.C. -1978- Notas sobre Juncus fontanesii Gay en la Península Ibérica. Rev. Fac. Ci. Univ. Oviedo 17-19: 323-332.

FUENTE, V. de la, E. ORTÚÑEZ \& L.M. FERRERO -1997- Contribución al conocimiento del género Festuca L. (Poaceae) en el País Vasco y Sistema Ibérico septentrional (Península Ibérica). Itinera Geobotanica 10: 317-351.

LAIINZ, M. -1960-Aportaciones al conocimiento de la flora cántabro-astur IV. Bol. Inst. Estud. Asturianos Supl. Ci. 1: 3-42.

LLAMAS, F. \& J. ANDRÉS -1983- Fritillaria legionensis Llamas \& Andrés, sp. nov. Lagascalia 11(1): 67-70.

LÓPEZ GONZÁLEZ, G. in CASTROVIEJO, S., (coord.) -1986- Flora Iberica. Plantas Vasculares de la Península Ibérica e Islas Baleares. Vol. I. Real Jardín Botánico, C.S.I.C. Madrid.

LOSA ESPAÑA, T.M. -1942- Plantas de Alar del Rey, Cervera de Pisuerga y otros puntos de la provincia de Palencia. Bol. Univ. Santiago 34: 3-38.

LOSA ESPAÑA, T.M. -1957- Catálogo de las plantas que se encuentran en los montes palentino-leoneses. Anales Inst. Bot. Cavanilles 15: 243-376.

MONASTERIO-HUELIN, E. in CASTROVIEJO, S., (coord.) -1998- Flora Iberica. Plantas Vasculares de la Península Ibérica e Islas Baleares. Vol. VI. Real Jardín Botánico, C.S.I.C. Madrid.

MONTSERRAT, P. -1994- Las campañas botánicas del profesor Losa España en la Cordillera Cantábrica. Jornadas conmemorativas del $1^{e r}$ 
centenario del nacimiento del Prof. T.M. Losa España. Granada.

NAVA, H.S. -1988- Flora y vegetación orófila de los Picos de Europa. Ruizia 6.

NAVARRO SÁNCHEZ, G. -1986-Vegetación y flora de las Sierras de Urbión, Neila y Cabrejas. Tesis Doctoral. Fac. Farmacia. Univ. Complutense. Madrid.

RIVAS-MARTÍNEZ, S., T.E. DÍAZ GONZÁLEZ, F. FERNÁNDEZ-GONZÁLEZ, J. IZCO, J. LOIDI, M. LOUSA \& A. PENAS -2002Vascular plant communities of Spain and Portugal. Addenda to the syntaxonomical checklist of 2001. Itinera Geobotanica 15 (2): 433-922.

VV. AA. -2000- Lista Roja de la Flora Vascular
Española (valoración según categorías UICN). Conservación Vegetal 6 (extra): 1-38. WILLKOMM, M. \& J. LANGE -1861-1880Prodromus Florae Hispanicae. 3 Vol. E. Scheweizerbart. Stuttgart.

Dirección de las autoras. 'Área de Botánica. Dpto. de Biodiversidad y Gestión Ambiental. Fac. de Ciencias Biológicas y Ambientales. Universidad de León. Campus de Vegazana. 24071 León. CRAI-Experimental. Herbario LEB Fac. de Ciencias Biológicas y Ambientales. Universidad de León. Campus de Vegazana. 24071 León. España. ${ }^{3}$ Indurot. Universidad de Oviedo. 33600 Mieres. Asturias.

*Autora para correspondencia: megarg@unileon.es

175. NOTAS COROLÓGICAS DEL MACROFITOBENTOS DE ANDALUCÍA (ESPAÑA). VII

\author{
María ALTAMIRANO, Eva LÓPEZ-BLANCO y Raquel ORRIACH
}

Recibido el 10 de enero de 2008, aceptado para su publicación el 21 de enero de 2008

Publicado «on line» en enero de 2008

New records for the macrophytobenthos of Andalusia (Spain). VII.

Palabras clave. Andalucía, corología, Lomentaria catenata, macroalgas marinas, Polisyphonia stricta

Key words. Andalusia, geographical distribution, Lomentaria catenata, Polisyphonia stricta, seaweeds

El presente trabajo añade nuevas especies (Conde et al., 1996b), Phaeophyceae (Floresa los catálogos de algas Rhodophyceae

Moya et al., 1995a) y Chlorophyceae

El trabajo presentado se enmarca en los proyectos "Estudio de la Flora Ficológica Andaluza", financiado por la Consejería de Medio Ambiente de la Junta de Andalucía, y "Modelación de la biodiversidad en Andalucía en un escenario de cambio en el uso del suelo y clima" (P05-RNM00935), financiado por la Consejería de Innovación, Ciencia y Empresa, de la Junta de Andalucía. 